\title{
Comprehensive Methods of Evaluation of Distance Learning System Functioning
}

\author{
Oleg Barabash ${ }^{1}$ \\ ${ }^{1}$ National Technical University of Ukraine "Igor Sikorsky Kyiv Polytechnic Institute", Kyiv, Ukraine. \\ E-mail: bar64@ukr.net
}

\author{
Oleksandr Laptiev ${ }^{2}$, Valentyn Sobchuk ${ }^{3}$, Ivanna Salanda ${ }^{4}$, Yulia Melnychuk $^{5}$ and Valerii Lishchyna ${ }^{6}$ \\ ${ }^{2,3}$ State University of Telecommunications, Kyiv,Ukraine. \\ ${ }^{3}$ National Defence University of Ukraine name after Ivan Cherniakhovskyi, Kyiv,Ukraine. \\ 4 Ternopil Regional Council Taras Shevchenko Regional Humanitarian-Pedagogical Academy of Kremenets, \\ Kremenets, Ukraine. \\ ${ }_{5,6}$ Lutsk National Technical University, Lutsk, Ukraine. \\ E-mail: alaptev64@ukr.net, v.v.sobchuk@gmail.com, salanda.ivanna@gmail.com, juliettathebest@gmail.com, \\ lvaleriy@gmail.com
}

Received: 22 January 2021; Accepted: 07 March 2021; Published: 08 June 2021

\begin{abstract}
Given recent events, training during quarantine can only take place remotely. To ensure quality training, communication must be seamless. To do this, the network must function smoothly. The solution to this problem is functionally stable networks that allow uninterrupted transmission of information due to redundancy. An important issue is the definition of redundancy. To solve this problem, the article considers the method of synthesis of the structure of the distance learning system. The method of synthesis of network structure used for providing distance learning by the criterion of maximum functional stability on the basis of the introduction of correcting communication lines is offered. With this method, you can develop tools for self-recovery of distributed software, taking into account the characteristics of disparate computer resources through the use of redundancy. This will allow you to develop functionally stable software systems, significantly reduce the recovery time of such systems after or in the event of possible failures. To increase the efficiency of the developed method, the mathematical model of the hyper network based on two hypergraphs was improved, which allows taking into account different requirements for the quality of the network.
\end{abstract}

Index Terms: Synthesize, Model, Network, Functional stability, Redundancy, Software systems.

\section{Introduction}

Modern software systems are created and operate in hardware platforms and operating systems that are constantly evolving. Most of them have a distributed structure, and the software components of the system are located on remote heterogeneous computing resources. Given recent events, training during quarantine can only take place remotely. To ensure quality training, communication must be seamless. Sooner or later, one of the programs, computing resources, communication equipment, or one of the available services will not cope with the increased load. Difficulties arise in the operation of such systems due to the increase in the number of objects and the integration of disparate systems into one disparate information network. In some cases, there is a possibility of external influence on the performance of software components. In all these cases, there is a problem with software recovery [1]. It is necessary to create a system that will operate under the influence of interference. To do this, the network must function smoothly.

Therefore, the problem of ensuring the smooth functioning of the distance learning system is very important.

The solution to this problem is functionally stable networks that allow uninterrupted transmission of information due to redundancy.

Therefore, the definition of redundancy is an important issue. The proposed system will provide proper communication during distance learning.

These networks function reliably under the influence of interference due to redundancy.

Redundancy consists of nodes and communication lines of the network. To determine the optimal redundancy, a method of network structure synthesis is proposed. To determine the optimal redundancy, a method of network structure synthesis is proposed.

The Synthesis procedure is based on the existing structure to which additional communication lines are added. As 
a result of synthesis the new structure in which the set of knots remains old, and communication lines become more will be received. Saturation of the structure with communication lines allows increasing the redundancy of the system. Which will further ensure the functional stability of the system.

This method allows you to set the optimal redundancy. The costs will be minimal. This system will provide proper communication during distance learning.

\section{Literature Review}

Self-healing systems can be divided into three levels, depending on the type of resources we track and influence: application software, system software and hardware level [1 - 4].

Self-recovery at the software application level is the ability of an individual application, software system or service to restore performance from within $[5,6]$.

System-level self-recovery applies to all operating system-level utilities and applications, regardless of their internal components. This is a type of self-recovery that we can develop at the level of the entire computer system. An example of a system-level solution is to restart a software process if it fails.

If a hardware failure occurs, the self-recovery is to find the workstations to which the software components are redistributed with the establishment of new network connections. As at the system level, it is necessary to periodically check the condition of various components of the equipment and act accordingly [7,16,29].

Particular attention to these issues exists in researchers of the properties of functional stability of complex technical systems that are able to self-organize under the influence of destabilizing factors $[8,9,28]$.

In $[10,11]$, a general strategy for ensuring the properties of functional stability for complex technical systems is developed, which is based on the implementation of three principles proposed by Professor Oleh Mashkov: detection of an emergency situation; localization of emergency situation; restoration of network operation due to redistribution of resources.

In $[12,19,21]$ the authors analyzed the mechanisms of self-organization of information heterogeneous networks and proposed new indicators and criteria for determining functionally stable networks in accordance with the concept of SON, and developed methods of self-organization of information network based on redistribution of resources between nodes that remain operational. However, the issue of identifying faulty components in the work is not paid attention.

The work $[13,15,27]$ is devoted to solving the problem of ensuring functional stability on the basis of Petri nets. In the works [14-17,24] network technologies of visual information transmission and development of information technology of task distribution for network systems using the simulation environment GRASS are considered. The methods mentioned in these works also use the mechanisms of network restructuring during anomalous overloads. At the same time, the issue of introducing redundancy (the issue of determining the optimal redundancy) remains open and unexplored.

Formulation of the problem.

In software systems, the term «self-healing» implies the presence of any application, service or system that can detect that it is not working properly, and without any human intervention to make the necessary changes to restore itself to normal or design condition $[18,20,23]$. The same actions can be performed by the system in case of probable failure of its structural elements $[5,19,26]$. Self-recovery is to make the system capable of making decisions by constantly checking and optimizing its condition and automatically adapting to changing conditions. The challenge is to make a functionally robust system that is able to respond to software and hardware changes and recover from failures or take appropriate action in the event of a failure. Ensuring functional stability is carried out by introducing structural, hardware, software and time redundancy. To do this, determine the optimal (by the criterion of maximum functional stability) network structure, which has an excessive number of communication lines in order to create alternative routes.

\subsection{The aim of the study}

Therefore, the aim of the study is to increase the redundancy of the system through the introduction of corrective communication lines, through which you can develop tools for self-recovery of information systems, taking into account the diversity of network objects. This will allow you to design functionally stable information systems, significantly reduce the recovery time of such systems in case of possible failures

\section{Methodology for Evaluation of the Efficiency of the Functioning of the Distance Learning System}

\subsection{Heterogeneous information network model}

A mathematical model that makes it possible to describe network processes, namely the processes of restructuring, self-recovery and self-tuning in the conditions of heterogeneous computer resources, is a hypernetwork.

Formally, an abstract hypernet can be described by the six $A S=(X, V, R ; P, F, W)$, which includes the following objects: 
- $X=\left(x_{1}, x_{2}, \ldots, x_{n}\right)-$ number of vertices;

- $V=\left(v_{1}, v_{2}, \ldots, v_{g}\right)$ - number of branches;

- $R=\left(r_{1}, r_{2}, \ldots, r_{m}\right)-$ number of ribs;

- $P: V \rightarrow 2^{X}$ is a map that corresponds to each element $v \in V$ the set $P(v)$ with $X$ of its vertices. Thus, the map $P$ determines the hypergraph $P S=(X, V ; P)$;

- F: R $\rightarrow \quad$ - a map that corresponds to each element $\mathrm{r} R$ the set F(r) of its branches, and the family of subsets of branches contains such subsets, the branches of which form a connected part of the hypergraph PS; the map F determines the hypergraph $\mathrm{FS}=(\mathrm{V}, \mathrm{R} ; \mathrm{F})$;

- $W: r \rightarrow 2^{P(F(r))} \forall r \in R$ - mapping that compares each element $r \in R$ subset $W(r)$ with $P(F(r))$ its vertices, where $P(F(r))$ is the set of vertices in $P S$, incident branches $F(r)$ with $V$. Thus, the map $W$ determines the hypergraph $W S=(X$, $R ; W)$.

Let the hypergraphs $P S=(X, V ; P)$ and $W S=(Y, R ; W)$ be given, then the mapping defines the abstract hypernetwork $A S=(P S, W S ; \Phi)$, which forms the connected part of the hypergraph $P S$.

It follows that pairs of hypergraphs $P S$ and WS can correspond to different abstract hypernets. In the future we will use different designations of hypernets where it will be convenient.

In figure 1 between the vertices $x_{1}$ and $x_{5}$ there is no pair of chains independent of the vertices, and yet the vertices $x_{1}$ and $x_{5}$ of this hypernetwork are externally 2 -connected, i.e. the external removal of any pair of vertices from the set $\left\{x_{2}, x_{3}, x_{4}\right\}$ destroys all circuits connecting the vertices $x_{1}$ and $x_{5}$.

The connectedness $\omega=\omega(S)$ of the hypernetwork $S$ is the smallest number of vertices, the removal of which leads to an incoherent hypernetwork as in figure 1;

The $\omega$-connectivity of the hypernetwork $S$ cannot be calculated by known methods of graph theory.

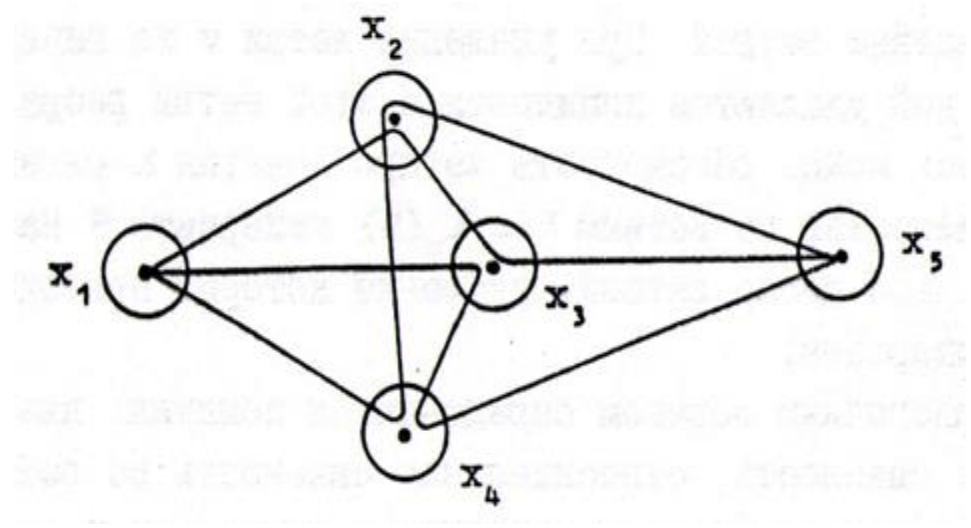

Fig.1. Hypernetwork model

3.2. A method of synthesizing the structure of the network by the criterion of maximum functional stability based on the introduction of corrective communication lines

Let the graphs of the primary network $P S=(X, Y)$ and the secondary network $W S=(Y, R)$ be known. Let's mark:

$\rho\left(v_{i j}\right)$ or $\rho P S\left(v_{i j}\right)$ - branch length $v_{i j} \in V$;

$\rho\left(r_{i j}\right)$ or $\rho_{W S}\left(r_{i j}\right)$ - rib length $r_{i j} \in R$;

$\partial\left(v_{i j}\right)$ - capacity of the branch $v_{i j} \in V$;

$\partial(r i j)$ - capacity of the rib $r_{i j} \in R$;

$\omega(S)$ - vertex hypernet connectivity $S$.

You need to build a hypernet $S=(P S ; W S ; \Phi)$, for which the conditions are met:

$$
\begin{gathered}
k \leq \omega(S) \rightarrow \max \\
\forall v \in V: \sum_{r \in \Phi^{-1}} \partial(r) \leq \partial(v)
\end{gathered}
$$


and which minimizes some functionality $\varphi(S)$ - the cost of design and operation of the network $S$.

The solution of this problem is an AI algorithm that searches for some valid hypernetwork that satisfies the criterion of maximum functional stability described above.3.3. AI Algorithm

Step 1. If $\omega(P S) \geq k$ and $\omega(W S) \geq k$, then go to step 2, else to step 15 .

Step 2. Between all pairs of vertices $x_{i}, x_{j} \in X$ find the maximum flows in the graphs $P S$ and WS, i.e. calculate $\mu_{P S}\left(x_{i}, x_{j}\right)$ and $\mu_{\mathrm{WS}}\left(x_{i}, x_{j}\right)$. If there is a pair of vertices $x_{i}, x_{j}$, for which $\mu_{\mathrm{PS}}\left(x_{i}, x_{j}\right)<\mu_{\mathrm{WS}}\left(x_{i}, x_{j}\right)$, then go to step 15 , else to step 3.

Step 3. Implement the ribs of the graph WS by the shortest path in the graph $P S$. If condition (1) is satisfied, then go to step 8, else to step 4.

Step 4. For all branches $v_{l t}$ with the maximum value of $\Delta_{l t}=\sum_{r \in \Phi^{-1}\left(v_{l t}\right)} \partial(r) / \partial\left(v_{l t}\right)$. If for some branches $v_{l t}, \Delta_{l t}>1$, then it is called supersaturated.

Step 5. Among the supersaturated branches choose a branch $v_{l t}$ with a maximum value of $\Delta_{l t}$ and find the rib $r=(x, y) \in \Phi^{-1}\left(v_{l t}\right)$ with a minimum value of $\partial(r)$. For each branch $v_{i j}$ calculate the value of $\delta_{i j}$ by the formula

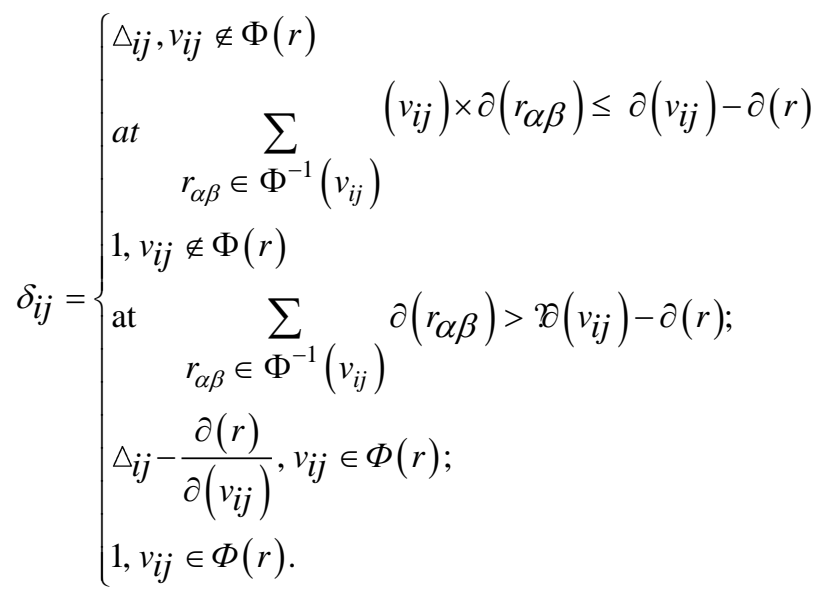

Similarly, for each branch $v_{i j}$ we set $\rho^{*}\left(v_{i j}\right)=\rho\left(v_{i j}\right) /\left(1-\delta_{i j}\right)$. If $\delta_{i j}=1$ then it is obvious that $\rho^{*}\left(v_{i j}\right)=\infty$.

Step 6. Choose the shortest path between the vertices $x, y$ in the graph $P S$ with the weight of the branches $\rho^{*}(v)$. If this route has a finite weight, then go to step 7, else to step 15 .

Step 7. Shake the rib r along the shortest route found in step 6. Recalculate all $\Delta_{l t}$ by the formula:

$$
\Delta_{l t}=\sum_{r_{\alpha \beta} \in \Phi^{-1}\left(v_{l t}\right)} \frac{\partial(r)}{\partial\left(v_{l t}\right)} .
$$

If there are no supersaturated branches in the resulting hypernet, then go to step 8 , else to step 5 .

Step 8 . If $\omega(s) \geq k$, then go to step 14 , else to step 9 .

Step 9. Find the minimum cross section $\left\{x_{1}, \ldots, x_{p}\right\} p<k$ of the hypernetwork behind the vertices.

Step 10. Find all ribs $r$, the paths of which contain vertices from the section $\quad\left\{x_{1}, \ldots, x_{\mathrm{p}}\right\}$, and the final vertices lie in different components of connectivity $S_{1}$ and $S_{2}$ of the hypernetwork $S \backslash\left\{x_{1}, \ldots, x_{p}\right\}$. At least one such rib will be found, since WS is $k$-linked. Choose among them the rib $r=(x, y)$ with the largest value of $\partial(r)$.

Step 11. For each branch $v_{i j}$ calculate $\delta_{i j}$ by the formula:

$$
\delta_{i j}\left\{\begin{array}{c}
1, \text { if } v_{i j} \notin \Phi(r) \text { and } \\
\sum_{r^{\prime} \in \Phi^{-1}\left(v_{i j}\right)} \partial\left(r^{\prime}\right)>\left(v_{i j}\right)-\partial(r) \\
\frac{1}{\partial\left(v_{i j}\right)} \sum_{r^{\prime} \in \Phi^{-1}\left(v_{i j}\right)} \partial\left(r^{\prime}\right), \text { in all other cases }
\end{array}\right.
$$

Similarly, for each branch $v_{\mathrm{ij}}$ we put $\rho^{*}\left(v_{i j}\right)=\rho\left(v_{i j}\right) /\left(1-\delta_{i j}\right)$. If $\delta_{i j}=1$, then $\rho^{*}\left(v_{i j}\right)=\infty$. 
Step 12. Find the shortest route between the vertices $x, y$ in the graph $\operatorname{PS} /\left\{x_{1}, \ldots, x_{p}\right\}$ with the weight of the branches $\rho^{*}(v)$. If this route has a finite weight, then go to step 13, else step 15 .

Step 13. We shake the rib $r$ along the shortest route found in step 12. If all ribs whose paths contain vertices from the cross section $\left\{x_{1}, \ldots, x_{p}\right\}$ are exhausted, and the end vertices lie in different connectivity components $S_{1}$ and $S_{2}$ of the hypernetwork $S \backslash\left\{x_{1}, \ldots, x_{p}\right\}$, then go to step 9, else in step 10. Repeat steps 9-13 until a hypernet is found that satisfies conditions (1), (2), or until the minimum cross section is repeated. In the first case, go to step 14, in the second - to step 15.

Step 14. A hypernetwork that satisfies the criterion of maximum functional stability (1)-(2) is found.

Step 15. Valid result not found.

The paper also considers the method of synthesis of the hypernetwork $S$, for which $\omega(S)=\omega(P S)$. This can be achieved by inserting correction ribs (communication lines) into the structure of the graph WS.

Let the primary network $P S=(X, V)$ and the secondary network $W S=(Y, R)$. It is necessary to find a hypernetwork $\mathrm{S}=\left(\mathrm{PS}, \mathrm{WS} S^{\prime}, \Phi\right)$ such that $\omega(S)=k \leq \omega(P S)$ and condition (2) is fulfilled, where WS ${ }^{`}(\mathrm{X}, \mathrm{R} \cup \mathrm{U})$, and $\mathrm{U}$ is the set of correcting ribs. And at addition of the next correcting rib of capacity of ribs WS`are listed.

\subsection{AII Algorithm}

Step 1. Implement the AI algorithm. If the resulting hypernet $\mathrm{S}$ satisfies condition (2) and $\omega(\mathrm{S})=\mathrm{k}$, then go to step 5, else to step 2 .

Step 2. If the hypernetwork $S$ does not satisfy condition (2), then go to step 4, else to step 3.

Step 3. In $\mathrm{S}$ find the minimum cross section of $\left\{\mathrm{x}_{i}\right\}$. Select in $\mathrm{S} \backslash\left\{\mathrm{x}_{i}\right\}$ the components of the connection $W S^{1}$ and $W S^{2}$. Find in $W S^{1}$ and $W S^{2}$ a pair of the nearest nonadjacent vertices $x_{1} \in W S^{1}$ and $x_{t} \in W S^{2}$. Connect the vertices $x_{1} \in W S^{1}$ and $x_{t} \in W S^{2}$ with an rib. List the capacities of the ribs using a given procedure for calculating the capacities. Go to step 1 .

Step 4. There is no valid solution.

Step 5. Hypernetwork $\mathrm{S}$ is found.

The convergence of the algorithm is obvious. The absence of a permissible solution is determined only by condition (2).

The paper also considers a fast algorithm for the synthesis of a hypernetwork with a fairly high connectivity.

Let the primary network $\mathrm{PS}=(\mathrm{X}, \mathrm{V})$ and the secondary network $\mathrm{WS}=(\mathrm{Y}, R)$ be given. You need to find a hypernetwork $\mathrm{S}=(\mathrm{PS}, W S ; \Phi)$ with the maximum possible connectivity. The weight of each branch and top is 1 .

\subsection{AIII Algorithm}

Step 1. Arrange the ribs $r=\left(\mathrm{x}_{j}, \mathrm{x}_{j}\right) \in \mathrm{R}$ in descending order of the distance $\rho=\left(\mathrm{x}_{j}, \mathrm{x}_{j}\right)$ in PS. We obtain a list of ribs $\left\{\mathrm{r}_{i}\right\}, i=1,2, \ldots, \mathrm{m} ; \mathrm{i}:=1$.

Step 2. Implement $r_{i}$ in PS by the shortest path (the shortest in total weights of vertices and ribs).

Step 3. Increase the weight of the vertices and ribs of the found route of the rib $r_{i}$. The weight increases by a certain amount of $z(S)$, which depends on the current state of the hypernetwork $\mathrm{S}$.

Step 4. If all ribs WS are implemented (if $\omega(\mathrm{S})=\min (\omega(\mathrm{PS}), \omega(\mathrm{WS}))$ ), then go to step 10, else to step 5), else $\mathrm{i}:=i+1$, to step 2 .

Step 5. Find the minimum cross section along the vertices of $\left\{\mathrm{x}_{1}\right\}$.

Step 6. Find all ribs $u$ that are weakly incidental to vertices with $\left\{\mathrm{x}_{1}\right\}$, such that their end vertices lie in different components of $S_{1}$ and $S_{2}$ connectivity of the $S \backslash\left\{\mathrm{x}_{1}\right\}$ hypernetwork. This, at least one rib is bound to be found, since WS is $k$-connected.

Step 7. In the primary network PS $\backslash\left\{\mathrm{x}_{1}\right\}$ between all pairs of end vertices $\mathrm{x}_{i}, \mathrm{x}_{j}$ we find the shortest routes. (The route will definitely be found since $\omega(\mathrm{PS}) \geq k$ ).Step 8. Choose the following pair $\mathrm{x}_{i}$ and $\mathrm{x}_{j}$, for which the following difference is minimal: $\rho_{P S \backslash\left\{\mathrm{x}_{1}\right\}}\left(\mathrm{x}_{i}, \mathrm{x}_{j}\right)-\rho_{P S}\left(\mathrm{x}_{i}, \mathrm{x}_{j}\right)$

Step 9. Implement the rib $\left(\mathrm{x}_{i}, \mathrm{x}_{j}\right)$ along the new path, then the connectivity of the components $S_{1}$ and $S_{2}$ increases. Repeat steps 3-6 until $\omega(\mathrm{S})$ connectivity increases, go to step 10 .

Step 10. A hypernet with quasi-maximum connectivity is found. The shortest path with adaptation method used in the algorithm allows to synthesize the initial structure of the hypernetwork $S$ with as much connectivity as possible due to the fact that in $S$ the number of weakly incident ribs on all vertices and branches is aligned. 
In figure 2 and figure 3 shows a non-synthesized and synthesized network structure with 12 switching nodes and 14 communication lines. The corresponding connected polynomials have the form

$$
\begin{aligned}
& P_{14}^{(1)}(p)=p^{14}+6 p^{13}(1-p)+ \\
& +15 p^{12}(1-p)^{2}+16 p^{11}(1-p)^{3}, \\
& P_{14}^{(2)}(p)=p^{14}+14 p^{13}(1-p)+ \\
& +81 p^{12}(1-p)^{2}+200 p^{11}(1-p)^{3},
\end{aligned}
$$

and their graphs are shown in figure 4.

The maximum effect from the optimization of the structure is achieved at point 0.869 , in which the difference between the values of the functional stability index reaches 0.467 . This fully confirms the thesis of the need to optimize network structures

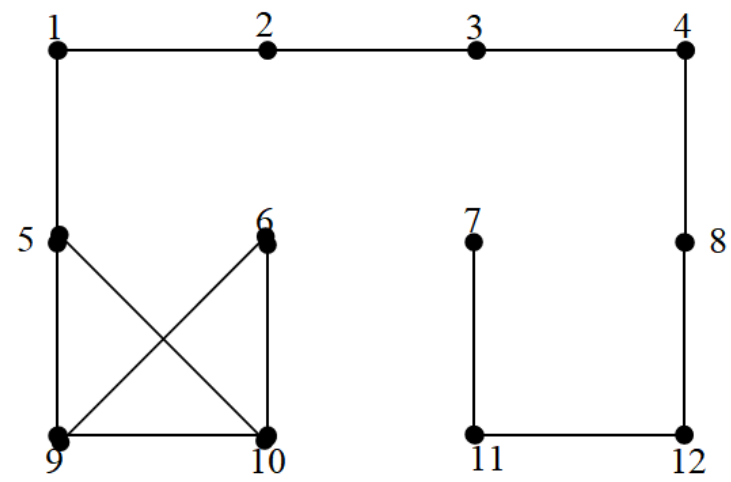

Fig.2. Min functionally stable G(12,14)-graph

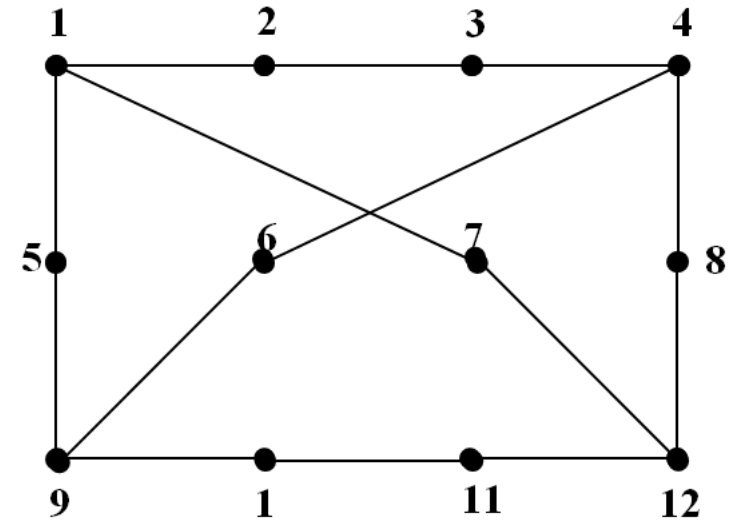

Fig.3.The max functionally stable $\mathrm{G}(12,14)$-graph.

\section{Experimental Confirmed Result}

Graphs of experimental and mathematical modeling of researches of a method of the analysis and synthesis of functional stability of branched information networks 


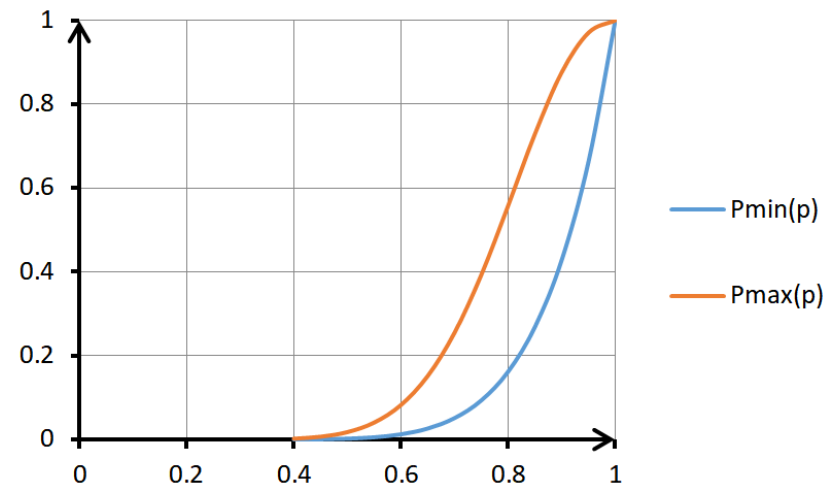

Fig.4. Graphs of polynomials of connectivity of graphs from figure 2 and figure 3.

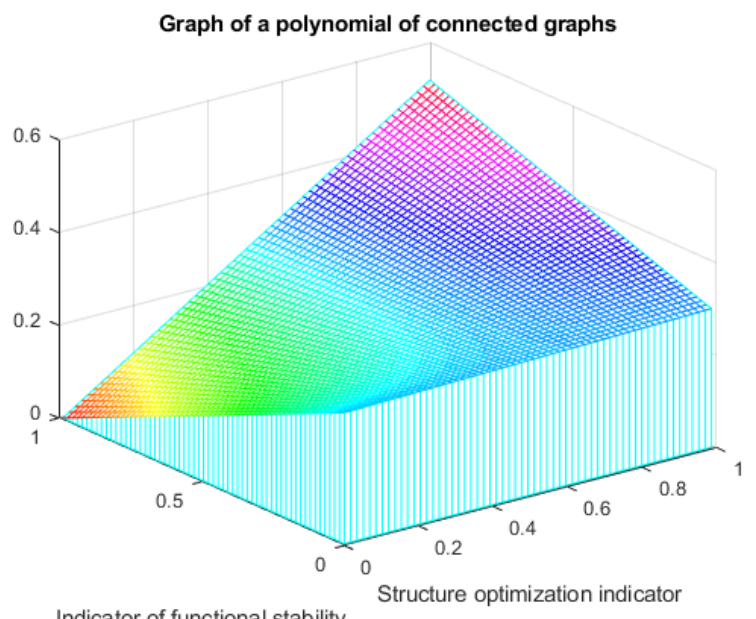

Fig.5. Graphs of polynomials of connectivity of graphs from figure 2.

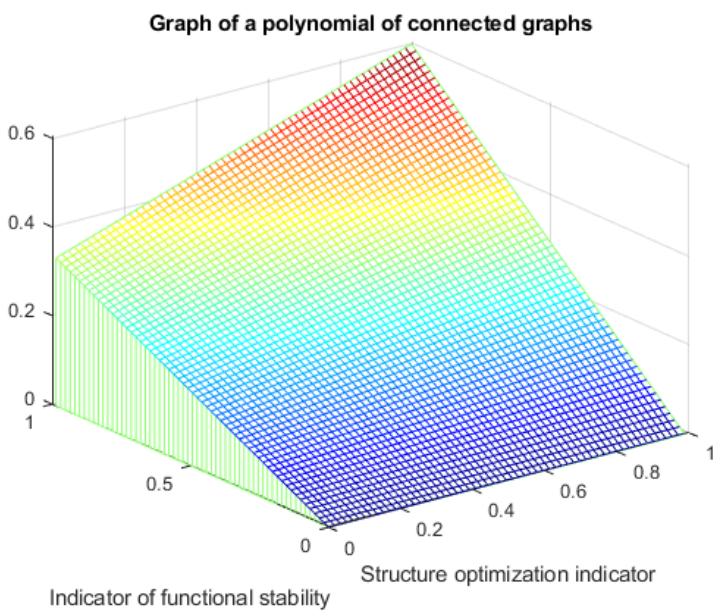

Fig.6. Graphs of polynomials of connectivity of graphs from figure 3.

From the three dimensional graphs obtained by modeling the results of the developed model, we see that the maximum effect of the structure optimization is achieved with the synthesized network structure.

The difference between the total values of functional stability reaches $12 \%$. This fully confirms the thesis of the need to optimize network structures.

Thus, the results of mathematical modeling investigated the validity of the developed methods of analysis and synthesis of functional stability of branched information networks. 


\section{Discussion of Experimental Results}

The proposed method increases the redundancy of the network. This method, in contrast to existing ones, aims to increase the redundancy of the network structure. The method of synthesis of network structure by the criterion of maximum functional stability on the basis of the introduction of correcting communication lines is offered. With this method, it is possible to develop tools for self-recovery of distributed software, taking into account the characteristics of disparate computer resources through the use of redundancy. This will allow to development of functionally stable software systems, significantly reduce the recovery time of such systems after or in case of possible failures. To increase the efficiency of the developed method, the mathematical model of the hyper network based on two hypergraphs was improved, which allows taking into account different requirements for the quality of the network.

An important limitation encountered in the operation of distance learning systems is the presence of interruptions caused by technological reasons when servicing under heavy load, or due to the effects of higher priority services. The results of the study show that the synthesized network has much greater connectivity. The maximum difference between the values of the indicator of functional stability reaches 0.467232 , which confirms the effectiveness of the developed method. This increases the network's resilience to interference (increases the number of new routes and increases redundancy.

The analysis of the obtained results allows calculating networks with full self-restoration of the functioning of the distance learning system. This method guarantees complete self-recovery of the distance learning system and does not require time to reboot and search and restore for routes. In this case, the system programs, or their individual components, are not idle. Thus, we can conclude that the purpose of the study is fully achieved.

The direction of further research may be to increase the redundancy of the network by optimizing the nodes of the network.

\section{Conclusion}

The paper proposes a method for synthesizing the structure of the network by the criterion of the maximum of the functional stability index based on the introduction of corrective communication lines. With this method, it is possible to develop tools for self-recovery of distributed software, taking into account the characteristics of heterogeneous computer resources through the use of redundancy.

This will allow you to design functionally stable software systems, significantly reduce the recovery time of such systems after or in the event of probable failures. To increase the efficiency of the developed method, the mathematical model of the hypernetwork based on two hypergraphs has been improved, which allows to take into account various requirements for the quality of network operation.

According to the simulation results of the proposed model, the maximum effect from the optimization of the structure is achieved at the point 0.869 relative units, in which the difference between the values of the functional stability indicator reaches 0.467 relative units. The difference between the total values of functional stability reaches $12 \%$. This fully confirms the thesis of the need to optimize network structures, and testifies to the reliability of the proposed method.

\section{References}

[1] Kravchenko Y. Evaluating the effectiveness of cloud services. 2019 IEEE 1th International Scientific-Practical Conference Problems of Infocommunications Science and Technology, PIC S\&T 2019, Kyiv, 2019, pp.120-124

[2] Pennington, J.; Socher, R.., Manning, C. D. Glove: Global vectors for word representation. EMNLP, 2014, vol. 14, pp. 153243.

[3] Dukhnovska K.K. Formuvannya Posukovy dynamical vector space.Shtunniy intertekt, 2016, no3.4, pp. 28-36.

[4] Barabash O.V., Open'ko P.V., Kopiika O.V., Shevchenko H.V., Dakhno N.B. Target Programming with Multicriterial Restrictions Application to the Defense Budget Optimization. Advances in Military Technology, 2019, vol. 14, no. 2, pp. 213 229.2019.

[5] Kreines M.G., Kreines E.M. Control model for the alignment of the quality assessment of scientific documents based on the analysis of content-related context. JCSSI, 2016, vol. 55, no. 6, pp. 938-947.

[6] Musienko A.P., Serdyuk A.S. Lebesgue-type inequalities for the de la Valée-Poussin sums on sets of analytic functions . Ukrainian Mathematical Journal September 2013, Volume 65, Issue 4, pp. 575 - 592.

[7] Musienko A.P., Serdyuk A.S. Lebesgue-type inequalities for the de la Vallée poussin sums on sets of entire functions . Ukrainian Mathematical Journal October 2013, Volume 65, Issue 5, pp. 709 - 722.

[8] Milov O., Yevseiev S., Milevskyi S., Ivanchenko Y., Nesterov O., Puchkov O., Yarovyi A., Salii A., Tiurin V., Timochko O. Development the model of the antagonistic agent's behavior under a cyber-conflict. Eastern European Journal of Advanced Technologies. Kharkiv.2019. 4/9 (100). pp. 6-19.

[9] Oleg Barabash, Oleksandr Laptiev, Oksana Kovtun, Olga Leshchenko, Kseniia Dukhnovska, Anatoliy Biehun. The Method dynavic TF-IDF. International Journal of Emerging Trends in Engineering Research (IJETER), Volume 8. No. 9, September 2020.pp 5713-5718 
[10] Barabash Oleg, Laptiev Oleksandr, Tkachev Volodymyr, Maystrov Oleksii, Krasikov Oleksandr, Polovinkin Igor. The Indirect method of obtaining Estimates of the Parameters of Radio Signals of covert means of obtaining Information. International Journal of Emerging Trends in Engineering Research (IJETER), Volume 8. No. 8, August 2020. Indexed- ISSN: 2278 - 3075. pp4133 - 4139

[11] Sweta Srivastav, Sangeeta Gupta. Results with Matlab coding of Middle Graph of Cycle and its related graphs in context of Sum Divisor Cordial International Journal of Innovative Technology and Exploring Engineering (IJITEE), Volume-8 Issue-2, February 2020. pp. 398-401.

[12] Mashkov O.A., Sobchuk V.V., Barabash O.V., Dakhno N.B., Shevchenko H.V., Maisak T.V. Improvement of variationalgradient method in dynamical systems of automated control for integro-differential models. Mathematical Modeling and Computing, 2019, Vol. 6, No. 2, pp. $344-357$.

[13] Barabash O., Dakhno N., Shevchenko H., Sobchuk V. Integro-Differential Models of Decision Support Systems for Controlling Unmanned Aerial Vehicles on the Basis of Modified Gradient Method. IEEE 5th International Conference on Methods and Systems of Navigation and Motion Control (MSNMC). 16-18 October, National Aviation University, 2018. Kyiv, Ukraine. pp. $94-97$.

[14] Ihor Ruban, Nataliia Bolohova, Vitalii Martovytskyi, Nataliia Lukova-Chuiko, Valentyn Lebediev. Method of sustainable detection of augmented reality markers by changing deconvolution. International Journal of Advanced Trends in Computer Science and Engineering (IJATCSE).Volume 9, No.2, March-April 2020, pp.1113 - 1120.

[15] Serhii Yevseiev, Oleksandr Laptiev, Sergii Lazarenko, Anna Korchenko, Iryna Manzhul. Modeling the protection of personal data from trust and the amount of information on social networks. Number 1 (2021), «EUREKA: Physics and Engineering» pp.24-31

[16] Rakushev, M., Permiakov, O., Tarasenko, S., Kovbasiuk, S., Kravchenko, Y. and Lavrinchuk, O. Numerical Method of Integration on the Basis of Multidimensional Differential-Taylor Transformations. In Proceedings of the IEEE International Scientific-Practical Conference Problems of Infocommunications Science and Technology, PIC S\&T`2019 - Proceedings, pp. $675-678$.

[17] Valentyn Sobchuk, Volodymyr Pichkur, Oleg Barabash, Oleksandr Laptiev, Kovalchuk Igor, Amina Zidan. Algorithm of control of functionally stable manufacturing processes of enterprises. 2020 IEEE 2nd International Conference on Advanced Trends in Information Theory (IEEE ATIT 2020) Conference Proceedings Kyiv, Ukraine, November 25-27. pp.206 -211.

[18] Y. Kravchenko, O. Leshchenko, N. Dakhno, O. Trush, O. Makhovych. Evaluating the effectiveness of cloud services. IEEE International Conference on Advanced Trends in Information Theory, ATIT'2019 - Proceedings, pp.120 - 124.

[19] MaksymukO.V., SobchukV.V., Salanda I.P., Sachuk Yu.V. A system of indicators and criteria for evaluation of the level of functional stability of information heterogenic networks. Mathematical modeling and computing. 2020. Vol. 7, No. 2. pp. $285-289$.

[20] O. Barabash, V. Sobchuk, N. Lukova-Chuiko, A. Musienko. Application of Petri Networks for Support of Functional Stability of Information Systems. 2018 IEEE First International Conference on System Analysis \& Intelligent Computing (SAIC). 08-12 October, Igor Sikorsky Kyiv Polytechnic Institute, 2018. Kyiv, Ukraine. pp. 36 - 39.

[21] Oleksandr Laptiev, Oleh Stefurak, Igor Polovinkin, Oleg Barabash, Savchenko Vitalii, Olena Zelikovska. The method of improving the signal detection quality by accounting for interference. 2020 IEEE 2nd International Conference on Advanced Trends in Information Theory (IEEE ATIT 2020) Conference Proceedings Kyiv, Ukraine, November 25 - 27. pp.172 -176.

[22] Barabash Oleg, Laptiev Oleksandr, Tkachev Volodymyr, Maystrov Oleksii, Krasikov Oleksandr, Polovinkin Igor. The Indirect method of obtaining Estimates of the Parameters of Radio Signals of covert means of obtaining Information. International Journal of Emerging Trends in Engineering Research (IJETER), Volume 8. No. 8, August 2020. Indexed- ISSN: 2278 - 3075. pp. $4133-4139$

[23] Yevseiev, S., Korol, O., Kots, H. Construction of hybrid security systems based on the cryptocode structures and flawed codes. Eastern-European Journal of Enterprise Technologies, 2017, 4(9-88), pp. 4-21

[24] Evseev, S., Kotz, H., Korol, O. Analysis of the legal framework for the information security management system of the nsmep.Eastern-European Journal of Enterprise Technologies, 2015, 5(3), pp. 48-59

[25] Yevseiev, S., Tsyhanenko, O., Ivanchenko, S., ...Milov, O., Shmatko, O. Practical implementation of the Niederreiter modified crypto-code system on truncated elliptic codes.Eastern-European Journal of Enterprise Technologies, 2018, 6(4-96), pp. 24-31

[26] Smelyakov K., Chupryna A., Hvozdiev M., Sandrkin D., Martovytskyi V. Comparative efficiency analysis of gradational correction models of highly lighted image. 2019 IEEE International Scientific-Practical Conference Problems of Infocommunications, Science and Technology (PIC S\&T), 8-11 Oct. 2019, Kyiv, Ukraine. pp. 703 - 708.

[27] Y. Kravchenko, K. Herasymenko, V. Bondarenko, O. Trush, M. Tyshchenko, O. Starkova, "Model of Information Protection system database of the mobile terminals information system on the territory of Ukraine (ISPMTU)", IEEE International Scientific-Practical Conference Problems of Infocommunications Science and Technology, PIC S\&T'2020 - Proceedings, in press.

[28] Oleg Barabash, Andrii Musienko, Spartak Hohoniants, Oleksandr Laptiev, Oleg Salash, Yevgen Rudenko, Alla Klochko. Comprehensive Methods of Evaluation of Efficiency of Distance Learning System Functioning. International Journal of Computer Network and Information Security(IJCNIS), IJCNIS Vol. 13, No. 1, Feb. 2021. pp.16 -28, Pub. Date: 2021-02-08. DOI: $10.5815 /$ ijcnis.2021.01.02

[29] Oleg Barabash, Andrii Musienko, Spartak Hohoniants, Oleksand Laptiev, Oleg Salash, Yevgen Rudenko, Alla Klochko, "Comprehensive Methods of Evaluation of Efficiency of Distance Learning System Functioning", International Journal of Computer Network and Information Security(IJCNIS), Vol.13, No.1, pp.16-28, 2021. DOI: 10.5815/ijcnis.2021.01.02

[30] Mousumi Mitra, Aviroop Chowdhury, " A Modernized Voting System Using Fuzzy Logic and Blockchain Technology", International Journal of Modern Education and Computer Science(IJMECS), Vol.12, No.3, pp. 17-25, 2020.DOI: $10.5815 /$ ijmecs.2020.03.03

[31] Bharati Sinha, Awadhesh Kumar Singh, Poonam Saini, "A Failure Detector for Crash Recovery Systems in Cloud", International Journal of Information Technology and Computer Science(IJITCS), Vol.11, No.7, pp.9-16, 2019. DOI: 
10.5815/ijitcs.2019.07.02

[32] Ashutosh Kumar Tiwari, Sandeep Varma Nadimpalli, " New Fusion Algorithm Provides an Alternative Approach to Robotic Path Planning", International Journal of Information Engineering and Electronic Business(IJIEEB), Vol.12, No.3, pp. 1-7, 2020. DOI: $10.5815 /$ ijieeb.2020.03.01

\section{Authors' Profiles}

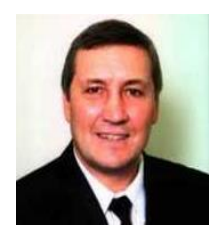

\section{Oleg Barabash}

Chair of high mathematics department of State University of Telecommunications, Ukraine, Kiev, Doct. of Sc. Born on July 28, 1964. M. Sc. (1986), PhD (1992), Doct. of Sc. (2006) from the National Academy of Defence of Ukraine; Assoc. Prof. (1996), Professor (2007) of computer systems department. Major interest: the functional stability of information systems and diagnostic systems for digital objects; security of distributed computer systems; design of the information security systems

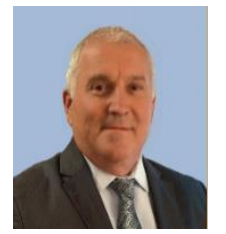

\section{Oleksandr Laptiev}

Doctor of Technical Sciences, Senior Researcher. Department of Information and Cybersecurity Systems. State University of Telecommunications Kyiv, Ukraine. Has an extensive practical experience in finding the means illegal obtaning of informations and transmitters. Areas of interest: information security, hidden transmitter search, radio intelligenceThe number of articles in national databases - 47. The number of articles in international databases -39 . H-index -4

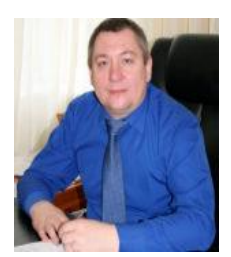

\section{Sobchuk Valentyn}

Doctor of Engineering, Professor Department of Higher Mathematics, Institute of Humanities and Natural Sciences, State University of Telecommunications : Study in Ukraine. Research interests: stability theory, functional stability of information systems of manufacturing enterprises, qualitative theory of nonlinear dynamical systems, differential equations Number of articles in national databases - 65. Number of articles in international databases -38 . H-index -4

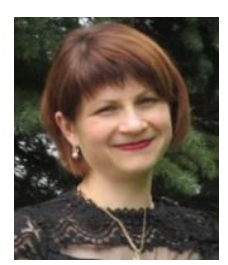

\section{Ivanna Salanda}

Candidate of Technical Sciences, Associate Professor, Department of Theory and Methods of Labor Education and Technology Ternopil Regional Council Taras Shevchenko Regional Humanitarian-Pedagogical Academy of Kremenets, Ternopil Region, Ukraine.The number of articles in national databases is 13. Number of articles in international databases - 1

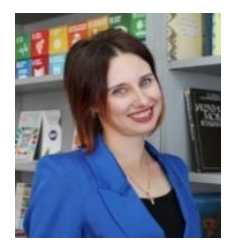

\section{Yulia Melnychuk}

Candidate of Pedagogical Sciences, Senior Lecturer, Department of Computer Science. Lutsk National Technical University. Lutsk, Ukraine. The number of articles in national databases is 14 . Number of articles in international databases - 11 .

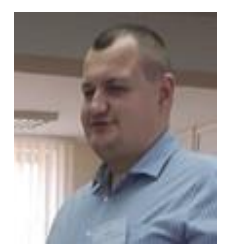

\section{Valerii Lishchyna}

Candidate of Technical Sciences, Associate Professor, Department of Computer Science. Lutsk National Technical University. Lutsk, Ukraine. The number of articles in national databases is 8 . Number of articles in international databases - 2 .

How to cite this paper: Oleg Barabash, Oleksandr Laptiev, Valentyn Sobchuk, Ivanna Salanda, Yulia Melnychuk, Valerii Lishchyna, "Comprehensive Methods of Evaluation of Distance Learning System Functioningt", International Journal of Computer Network and Information Security(IJCNIS), Vol.13, No.3, pp.62-71, 2021. DOI: 10.5815/ijcnis.2021.03.06 